\title{
Analogical transfer: From schematic pictures to problem solving
}

\author{
ZHE CHEN \\ University of Kentucky, Lexington, Kentucky
}

\begin{abstract}
A series of experiments was conducted to explore whether individuals can solve problems by transferring conceptual information gained from schematic pictures and to examine the mechanisms involved in this transfer process. Subjects viewed a schematic picture and then attempted to solve an insight problem to which the conceptual information from the picture could be applied. The results indicate that the degree of similarity-specifically, superficial and procedural similaritybetween a source schematic picture and the target problem determined transfer performance. Discussion focuses on the relationship between these two types of similarity and the two key cognitive components involved in transferring pictorial analogies to solve problems: accessing the pictorial analogues and executing the solutions.
\end{abstract}

The importance of analogy in problem solving has been repeatedly demonstrated, particularly during the past two decades. Analogical problem solving refers to the transfer of previously acquired knowledge or solutions from one context or domain to another. Analogous information can be displayed in various modalities such as verbal materials, real-world scenes, and pictorial format. A vast body of research has revealed the powerful facilitative effects of language-based analogies in problem solving (e.g., Brown \& Kane, 1988; Gentner, 1989; Gick \& Holyoak, 1980; Novick, 1988; Oppenheimer, 1956; Reed, 1987; Ross, 1989). Yet surprisingly little research has been done to determine whether and how individuals come to comprehend, retrieve, transform, and utilize pictorial information in solving analogous problems.

Visual analogues have long been considered an important source for scientific discoveries and everyday problem solving (see, e.g., Gordon, 1961, 1979; Krueger, 1981; Wertheimer, 1959). Theorists (e.g., Chafe, 1976; Kintsch, 1974; Larkin \& Simon, 1987; Levin, 1982; Mayer \& Gallini, 1990; Novick, 1990; Simon, 1981) have suggested that visual representations play a critical role in problem solving because they display structural relationships clearly and economically. Yet the facilitative function of pictorial materials for learning and problem solving remains to be explored, and critical questions arise concerning how individuals identify the conceptual infor-

I am grateful to Marvin Daehler, Nancy Myers, Brian Ross, Barry Stein, and several anonymous reviewers for their valuable comments on earlier versions of this manuscript. I would also like to thank Robert Lorch and Brian Ross for their advice on data analyses, Laura AndersonChen for her editing wisdom, and Michael Beck and Jennifer Wright for their assistance in data collection. Portions of this research were presented at the 33rd meeting of the Psychonomic Society in St. Louis, November 1992 Correspondence may be addressed to Z. Chen, Department of Psychology, University of Kentucky, Lexington, KY 40506-0044 (e-mail: zchen00@ukcc.uky.edu). mation from visual displays (e.g., realistic or schematic pictures) and use the conceptual meanings to guide subsequent problem solving. The present research focuses on problem solving by drawing analogies between a schematic picture and a target problem and using the conceptual meaning from the picture to generate appropriate solutions. A schematic pictorial analogue is a sequence of diagrammatic drawings in which potentially useful concepts or solutions are depicted through the spatial organization of sketches of geometric forms and objects.

\section{Previous Studies}

Recent studies have shown some inconsistent findings concerning the function of pictorial analogues on problem solving. Some schematic pictures can serve as powerful analogues that facilitate subsequent problem solving (see, e.g., Beveridge \& Parkins, 1987; Dreistadt, 1969), whereas others are insufficient (see, e.g., Gick, 1985). For example, Dreistadt asked subjects to solve a Tree Planting problem, in which subjects needed to figure out how to plant ten trees in five rows with four trees in each row. The solution is to plant the trees in the shape of a five-pointed star. The source pictures included a drawing of a starfish among other ordinary-looking fish in an aquarium and a drawing of a clown wearing a starshaped cap. Subjects who viewed the visual analogues were more able to come up with the correct solution for the Tree Planting problem.

Gick and Holyoak (1983; Gick, 1985) used Duncker's (1945) Radiation Problem as their target problem. A successful solution for this problem utilizes the principle of convergence. Several rays coming from different directions must converge on a single focal point in order to destroy a tumor while at the same time they do not destroy the surrounding healthy tissue. Before attempting to solve the problem, subjects were provided with a source story only, a diagram only, or a source story with a diagram at the end of the text. The source diagram schemat- 
ically depicted a potential analogue for the target problem and contained two parts: a large, thick arrow representing the strong force and smaller arrows converging to the center indicating the weaker forces from different orientations. Gick (1985) found that the combination of the analogous story with the diagram yielded a significant improvement in problem-solving performance. However, presenting the source diagram alone failed to facilitate transfer.

Beveridge and Parkins (1987) carried out further experiments with a similar paradigm in order to explore whether a more explicit depiction of the analogous solution would enable adults and children to solve the target problem more effectively. These researchers argued that the diagram was perhaps not seen as representing a general solution schema analogous to the target problem, because the diagram had been introduced as a visual pattern memory task. Moreover, the diagram did not depict some of the crucial aspects of the analogy, in that it did not unequivocally indicate the summative aspects of the solution. To that end, these researchers created a new diagram that "represented intensity and summation at the point of convergence" (Beveridge \& Parkins, 1987, p. 232). The results indicated that diagrams which sufficiently conveyed the nature of the convergence solution effectively facilitated problem solving.

The question of whether schematic pictures can serve as valid analogues in problem solving is an issue worthy of attention. Yet a variety of factors could influence the use of an analogue, and the reasons for the ineffectiveness (Gick, 1985) or the effectiveness (Beveridge \& Parkins, 1987) of visual analogues are far from completely understood. Hence, it would seem essential to examine the mechanisms involved in the transfer process. For example, why are some pictures or diagrams more facilitative in transfer than others? What properties of a picture make it a more useful, sufficient, and powerful analogue in problem solving? And how are these features associated with the cognitive processes involved in analogical transfer? The present research is the first step of a systematic investigation of the mechanisms involved in the transfer of schematic pictures in problem solving.

\section{Framework of the Present Research}

Researchers (e.g., Gentner, 1989; Holyoak, 1984) have proposed that four major cognitive components are involved in analogical transfer: (1) constructing a mental representation of the source and target problems (Brown, Kane, \& Echols, 1986; Chen \& Daehler, 1989); (2) noticing the potentially analogous relationship between the problems (Holyoak \& Koh, 1987; Ross, 1984); (3) mapping the correspondences between the key elements of the source and target problems (Gentner, 1989; Reed, 1987), and (4) applying (executing) a solution appropriate to the target problem (Novick \& Holyoak, 1991; Ross, 1987). The present research examined whether a schematic picture can serve as an effective source analogue in problem solving and focused on two key com- ponents-namely, the accessing and executing processes involved in transfer.

In a large number of problem-solving studies done with verbal source analogues, it has been demonstrated that one major obstacle to analogical transfer is the failure to access a potentially useful source analogue spontaneously (e.g., Brown \& Campione, 1981, 1984; Daehler \& Chen, 1993; Ross, 1984, 1987; Stein, Way, Benningfield, \& Hedgecough, 1986; Vosniadou, 1989). One factor promoting the accessing (retrieval) of source analogues is a high degree of surface similarity between the source and target problems. Subjects tend to transfer the source solutions readily once the source and target problems share some solution-irrelevant but salient details such as objects, characters, or other semantic features (see, e.g., Crisafi \& Brown, 1986; Holyoak, Junn, \& Billman, 1984; Perfetto, Bransford, \& Franks, 1983; Ross, 1984). Another factor influencing the accessing process involves the provision of hints that the subject should consider the source problem before attempting to solve the target (see, e.g., Gick \& Holyoak, 1980; Ross \& Kennedy, 1990; Weisberg, DiCamillo, \& Phillips, 1978).

Yet successful retrieval of the source analogue does not ensure that a solution principle can be automatically transformed into an operational procedure for a target problem. Another important process involves appropriately executing a solution principle in solving a concrete problem, a process that can be impeded if the source solution does not provide adequate or similar implementation details. Although researchers (e.g., Bassok, 1990; Ross, 1989; Ross \& Kennedy, 1990) have started to distinguish the executing process from other components, little is known about the factors influencing transfer via this process. Recently, Novick and Holyoak (1991) demonstrated that when numerical correspondences between source and target mathematical problems were clearly specified, subjects were more likely to transfer an appropriate mathematical procedure than when only conceptual relationships were provided. Novick and Holyoak reported that mapping the conceptual relations between the source and target problems did not guarantee successful transfer and suggested that another cause of difficulty in transferring solutions across problems is associated with the adaptation process, which requires matching the numerical correspondences between the source and target problems. Transfer was enhanced when the numerical mapping between the source and target problems was clearly explained.

In the present experiments, the importance of the accessing and executing processes were studied by manipulating two types of similarity-surface and procedural similarity - between a source schematic picture and a target problem. The first type of similarity involved superficial features shared by a source picture and the target problem. It was predicted that this type of similarity would aid the retrieval of an analogous source solution. A schematic picture might lack surface similarity to a target problem, and hence, the analogous information 
might be difficult to retrieve or access spontaneously when needed. Thus, adding drawings of concrete objects that were attributionally similar to those in the target problem should facilitate the accessing process and subsequent transfer.

The second type of similarity involved procedural details shared by the source picture and the required target solution. In the present research, procedure is defined as the transformation of a general solution principle or concept into concrete operations (a sequence of actions). Implementational similarity refers to the extent to which the procedural details illustrated in the pictorial schematics match or differ from those required for a given target problem. The major idea is that even if subjects are able to access the analogous source information spontaneously, they may still be faced with the problem of how the general solution principle can be applied or transformed into a workable procedure appropriate for solving the target problem. It was hypothesized that when the general solution principle between a source and a target problem was isomorphic but was accompanied by a discrepant implementation procedure, the dissimilar procedural details would create an obstacle for transferring the source solution principle. It was thus predicted that similarity of implementation procedure would facilitate the execution or utilization of a solution principle.

Thus, either surface or procedural similarity alone might not be sufficient for achieving transfer. On the one hand, although surface similarity might increase the probability of retrieving a potentially useful solution principle, it did not ensure that problem solvers would benefit from the pictorial analogue, because of potential obstacles in implementing the solution principle. On the other hand, even if the source picture and the target problem shared a similar solution procedure, transfer might not occur, because of a failure to access the source information. The possible interaction between these two types of similarity in analogical transfer was also the focus of the present research.

\section{GENERAL METHOD}

The tasks in this set of experiments involved transferring the conceptual information from a schematic picture in order to solve an analogous problem. The target problem involved "Weighing the Elephant" with a small scale (see Appendix A). The first step of the correct solution is to put the elephant on a boat and mark the water level on the boat. The elephant then is replaced with some smaller objects, such as rocks, until the water surface reaches the mark. The smaller objects are then weighed separately with the scale. The total weight of the smaller objects equals the elephant's weight.

This problem was created for the present research because, although it requires an insight (the weight equivalence principle: the combined weight of lighter objects can equal that of a heavy object), grasping this general concept, in and of itself, is not adequate for solving the problem. A complete solution requires extending the insight into a concrete procedure (the weight equalization procedure: equalizing the weights of the elephant and the smaller ob- jects). In this way, this target problem differs from other problems requiring other types of insight (see, e.g., Gick \& Holyoak, 1980; Holyoak \& Koh, 1987; Needham \& Begg, 1991; Ross, Ryan, \& Tenpenny, 1989) and well-defined problems such as math and physics tasks (see, e.g., Bassok \& Holyoak, 1989; Novick, 1988; Reed, 1987, 1989; Ross \& Kennedy, 1990). The requirement of both an insight principle and a procedural answer allows us to explore in more detail the processes involved in executing the relevant information from the source pictures in solving the target problem.

The general paradigm of the present research involved first showing subjects a schematic picture that provided potentially analogous information for the solution of the target problem. The source picture conveyed the weight equivalence principle by illustrating the phenomenon that several lighter objects can push down a compressible surface to the same degree as one heavy object can (see the examples in Figure 1). The relations (surface and procedural similarity) between a given source picture and the target problem were manipulated, and the corresponding variation in solving the target problem was observed and analyzed.

The present series of experiments addressed the issues of whether and how a schematic picture facilitates problem solving. Experiment 1 was a preliminary study in which we tested the overall effects of similarity between a schematic picture and the target problem on problem-solving performance. Experiment 2 was designed to examine the separate effects of surface and procedural similarity between a source pictorial analogue and the target problem on transfer. This study tested whether surface similarity enhances the accessing process and whether procedural similarity facilitates the executing process. Experiment 3 was conducted to explore whether the manipulation of procedural similarity affects subjects' comprehension of the weight equivalence principle and whether a distinction between surface and procedural similarity can be made. Finally, Experiment 4, with a hint/no-hint paradigm, was performed to further test the relations between procedural similarity and the executing process.

\section{EXPERIMENT 1}

The major purpose of this experiment was to investigate the effects of the overall similarity between the source picture and target problem on subsequent problem solving. For even if a pictorial analogue illustrates a potentially useful solution to a problem situation, the lack of surface (attributional) and procedural similarity between the source pictures and the target problem might cause difficulty in accessing and executing the relevant information. Thus, the primary objective of this experiment was to determine whether or not schematic pictures containing physical attributes (objects) and procedures either similar or dissimilar to the target problem affect the transfer of the source solution.

This experiment was also designed to determine whether item abstractness and concreteness and the degree of clarity of the conceptual relations illustrated in the schematic pictures influence transfer. It has been demonstrated that abstract verbal materials are less comprehensible than concrete verbal materials (see, e.g., Johnson, Bransford, Nyberg, \& Cleary, 1972; Pezdek \& Royer, 1974) and that abstract materials tend to be less efficient than concrete examples in facilitating problem solving (see, e.g., Anderson, Farrell, \& Sauers, 1984; Medin \& Ross, 
1989). We extended these findings to the domain of schematics and hypothesized that both the abstract nature of such schematic pictures and the implicitness of their depicted conceptual meanings would impede the encoding and transferring of the conceptual information. Adding concrete and common objects to the schematics would increase the ease with which the conceptual meaning of a picture is comprehended and, thus, enhance subsequent transfer.

Moreover, this experiment was an examination of whether the clarity of the conceptual meanings would depend on how the structural relations were depicted rather than on what objects were contained in a schematic picture. Previous research done with verbal analogical passages has demonstrated that representations of more manifest relational structures facilitate analogical accessing and mapping (e.g., Chen \& Daehler, 1992; Clement, 1994; Clement \& Gentner, 1991). In the present experiment, the effect of explicit conceptual relations on transfer was examined by manipulating the level of elaboration with which the weight equivalence principle was depicted in the schematics. It was predicted that if the vagueness of the conceptual relations illustrated in the schematics was indeed a hindrance to comprehension and transfer, an elaborated depiction of the principle would promote transfer performance.

\section{Method}

Subjects. One hundred and forty-three undergraduates enrolled in introductory psychology courses at the University of Kentucky participated in this experiment for course credit.

Materials. The target problem was the Weigh the Elephant problem, in which subjects were asked to generate possible solutions for obtaining the weight of an elephant. The story problem described a situation in which a boy needed to weigh an elephant but had only a small scale with which to do it. Some items (e.g., a small scale, a boat, and rocks) relevant to the generation of an appropriate solution were introduced naturally in the story, and sketches of these objects, along with several irrelevant items, were provided on the problem solution sheet (see Appendix A).

Before the presentation of the target problem, the subjects in the experimental conditions received schematic pictures illustrating conceptual information analogous to the solution for the target problem. The pictorial schematics depicted the concept that small objects can have the same weight as that of a big item and hence can push down a compressible surface to the same degree. The physical attributes of the objects in the pictures and the degree of clarity of the conceptual relations were manipulated. The subjects in the control condition received an irrelevant picture before being asked to solve the target problem.

Design. The target problem was identical across conditions. The conditions differed in the pictorial stimuli used as source analogues. A 4 (attributes: abstract, dissimilarity, low similarity, high similarity) $\times 2$ (conceptual relations: implicit, explicit) betweensubjects design plus a control group was used in this experiment. In each experimental condition, a source picture illustrating a solution analogous to that for the target problem was presented (see Figure 1). In the abstract condition, only abstract geometric shapes with no concrete objects were shown. The first diagram in this series was a platform composed of lines. The second diagram showed a solid square pressing down the platform, and the third depicted several small squares compressing the platform to the same degree as the big square did in the second diagram. In the dissimilarity schematic picture, concrete common objects were used. A sketch of a car replaced the big square, and TV sets replaced the small squares. Thus, concrete objects dissımilar to the key objects (elephant, rocks, and boat) in the target problem were included. In the low-similarity condition, the car and TV sets were replaced by sketches of a pig and cats, which were more simılar in category and in physical attributes to the elephant in the target problem.

The high-similarity pictures also contained the pig and cats but not the "compressible" line platform. Instead, the first diagram showed a container-like shape "floating" near the top of a pile of broken lines. The second diagram showed the container-like shape, now holding the pig, sunken farther into the broken lines. In the final diagram, the cats replaced the pig, and the container sank down to the same degree as in the second diagram. The way that the container-like shape sank into the broken lines was similar to the boat solution.

In the four implicit relations conditions, the relationship between the large object and the smaller items (i.e., they have the same weight) was not explicitly indicated. However, in each of the four explicit relations conditions, this relation was elaborated: additional diagrams showed the large item being equal to $(=)$ the smaller objects. The eight schematic pictures are presented in Figure 1 .

Procedure. The subjects were tested in groups of 4 to 15 . A booklet containing the source picture and the target problem was constructed. The first page was the informed consent, which notified subjects that they would be asked to view some visual stimuli and solve a hypothetical problem. The second page contained the schematic picture serving as the source analogue. The subjects in all groups were instructed to view the source picture, which was presented for $50 \mathrm{sec}$.

Approximately 2 min elapsed between the picture-viewing and the problem-solving phases. During this interval, the experimenter handed out experimental credit slips and asked each participant to sign one. The subjects were then instructed to turn to the third page, which contained the target story problem. The line drawings of the relevant and irrelevant items along with their labels (animal, rocks, small scale, trees, table, boat, containers, and boxes) were presented below the story. Space allowing for written solutions was also provided. Subjects were asked to read the story problem carefully within $100 \mathrm{sec}$ and then, using only the items illustrated on the page, to generate any solutions they thought appropriate. The constraint of using the illustrated items was imposed in order to elicit solutions relevant to the manipulations of the source schematic pictures. To estimate the length of time and the order of each solution generated, five sections of space divided by lines were provided. The subjects were told to write down their solutions in the first section and to continue their answer in the next section when a signal was given. The experimenter gave a signal to continue answers in the next section every $40 \mathrm{sec}$. Thus, a total of $200 \mathrm{sec}$ was allowed for generating solutions to the target problem.

A brief questionnaire was given after the problem-solving phase. The subjects were asked if they had seen the target problem before participating in the experiment. They were also asked whether the source picture had helped them solve the target problem and to indicate on a 7 -point scale how useful the picture had been in solving it.

Scoring. Two major measures concerning the success and readiness of problem solving were examined. The first was the percentage of subjects successfully solving the target problem. If the answer was correct and complete, a score of 1 was given. An appropriate and complete solution was one that included both the weight equivalence idea and the weight equalization procedures. If the answer was incorrect, inapproprate, or incomplete, a score of 0 was given. One example of an incorrect and inappropriate answer would be to "cut the elephant into pieces and then weigh 
Implicit Relations:

Abstract

Dissimilarity

Low Similarity

High Similarity

Explicit Relations:

Abstract

Dissimilarity

Low Similarity

High Similarity
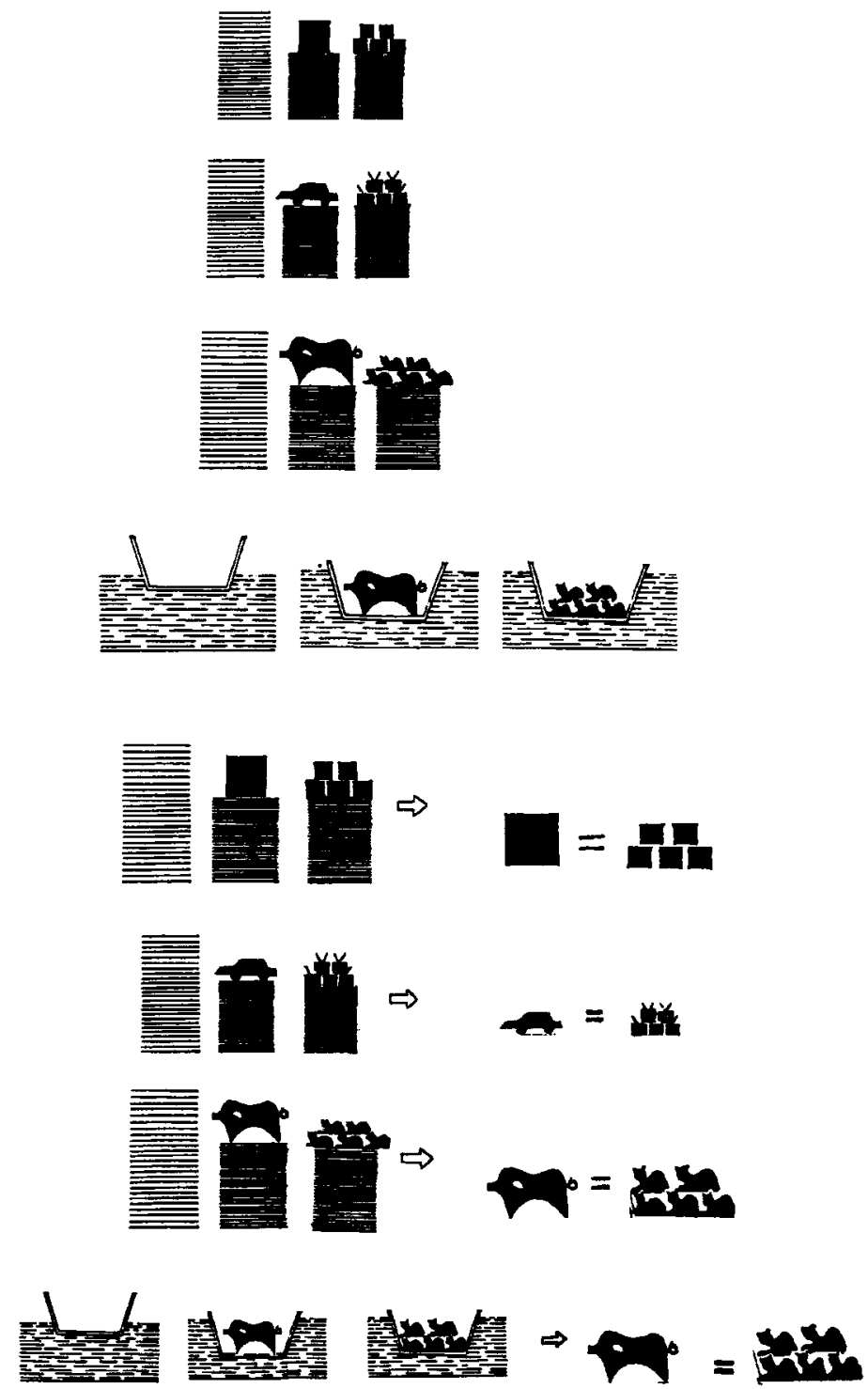

Figure 1. Schematic pictures used in Experiment 1.

them." An answer that contained the idea of using the smaller items without explaining why and how was also scored as 0 .

The second scoring for problem-solving performance was a 6point efficiency scale designed to evaluate how quickly and readily a correct answer was provided. ${ }^{1}$ If the correct answer occurred during the first time section, it received a score of 1 ; if during the second time section, the efficiency score was 2 ; and so forth. If no correct answer was generated, a score of 6 was assigned.

Two secondary measures were also included. One was concerned with the subjects' responses to the question of whether the source pictures had helped them solve the problem (yes/no). The other was concerned with how helpful the pictures were, and, in order to determine their perceived usefulness, subjects were asked to evaluate them on a 7-point scale ranging from 1 (not useful) to 7 (very useful).

All the data concerning the subjects' problem solving were coded by a scorer. In addition, the performance of 40 randomly selected subjects was independently scored by another observer for a reliability check. Agreement between the two scorers on the solutions (yes/no) was $97 \%$. The correlation between the two observers on the order (time section) in which the correct answers were provided was above .98 .

\section{Results}

As is indicated in Table 1, the frequency of problem solving was determined by the features of the source schematic pictures. We used an analysis of variance (ANOVA) on the frequency data (cf. Bevan, Denton, \& Myers, 1974; Hsu \& Feldt, 1969; Myers \& Well, 1991). A $2 \times 4$ ANOVA was performed, with conceptual relations (explicit, implicit) and attributes (abstract, dissimilarity, low similarity, high similarity) as the independent variables. This analysis revealed a main effect of attributes $\left[F(3,120)=4.95, M S_{\mathrm{e}}=.213, p<.005\right]$ and a mar- 


\begin{tabular}{|c|c|c|c|c|}
\hline Condition & $n$ & $\begin{array}{c}\% \text { Subjects } \\
\text { Solving Problem }\end{array}$ & $\begin{array}{c}\text { Usefulness } \\
(\%)\end{array}$ & $\begin{array}{c}\text { Usefulness } \\
\text { Rating }\end{array}$ \\
\hline \multicolumn{5}{|l|}{ Implicit Relations } \\
\hline Abstract & 16 & 13 & 40 & 2.9 \\
\hline Dissimilarity & 16 & 19 & 31 & 2.9 \\
\hline Low similarity & 16 & 25 & 50 & 3.1 \\
\hline High similarity & 16 & 56 & 44 & 3.7 \\
\hline \multicolumn{5}{|l|}{ Explicit Relations } \\
\hline Abstract & 16 & 31 & 44 & 3.0 \\
\hline Dissimilarity & 16 & 38 & 50 & 3.1 \\
\hline Low similarity & 16 & 38 & 44 & 3.3 \\
\hline High similarity & 16 & 69 & 63 & 3.6 \\
\hline Control & 15 & 7 & 27 & 2.3 \\
\hline
\end{tabular}

ginally significant main effect of conceptual relations $\left[F(1,120)=3.68, M S_{\mathrm{e}}=.213, p=.057\right]$. The interaction between these two factors was not significant. These patterns of performance show that, with the increase of attributional similarity between the source schematics and the target problem, subjects benefitted more from the source pictures. Furthermore, the more explicit conceptual relations facilitated the transfer of the analogous information. A Student-Newman-Keuls test revealed that subjects in the high-similarity conditions outperformed those in the control condition ( $p s<$ .05 ) and that subjects in the high-similarity, explicit relations condition performed better in solving the problem than those in the abstract, implicit relations condition $(p<.05)$ and the dissimilarity, implicit relations condition $(p<.05)$.

The percentage of subjects recognizing the usefulness of the source pictures and the usefulness ratings on the 7-point scale reveals a pattern similar to that of the problem-solving performance. However, no significant effects were obtained.

\section{Discussion}

Why was the basic abstract schematic picture insufficient to trigger transfer in problem solving? Several possible reasons for its ineffectiveness were examined in this experiment. First, an attempt was made to increase transfer performance by replacing the geometric shapes with sketches of concrete objects. The results reveal, however, that adding drawings of concrete items to the schematics did not improve transfer unless they were highly similar to the target problem. Second, as predicted, subjects receiving schematic pictures with explicit conceptual relations evidenced greater transfer than did those receiving pictures with implicit conceptual relations. The manifestation of the conceptual relations made it easier for solvers to extract the potentially useful conceptual meaning from the pictures, suggesting that the implicitness of the conceptual information in the pictorial schematics may be responsible for the low transfer performance. The explicitness of the conceptual relations depicted could have also enhanced retrieval of the analogous information when subjects were solving the target problem. Further clarification of these issues is needed, but it is beyond the scope of the present paper to do so.

The focus of the present experiment was on the effects of similarity on transfer. The results indicate that, in comparison with the dissimilarity and low-similarity pictures, the high-similarity schematics greatly enhanced subjects' problem-solving performance. This result appears to be consistent with previous findings obtained with verbal materials, which indicated that similar surface features between source and target problems facilitate transfer. However, Experiment 1 did not distinguish between the effects of attributional and procedural similarity. The high-similarity and other types of source pictures differed not only in the degree of surface similarity to the target problem but also in the level of procedural similarity. The weight equivalence principle was illustrated with different procedures. In the high-similarity pictures, while the objects similar to those in the target problem might have enhanced the accessing process, the procedural suggestion - that is, the container-like shape "floating" near the top of a pile of broken lines and then "sinking" into the broken lines-might have also facilitated the execution component. Because these two possible effects were not separated in Experiment 1, it is not yet clear whether the superior performance in the high-similarity condition should be attributed to the surface and/or the procedural similarity shared by the source picture and target problem. Experiment 2, therefore, was designed to distinguish between these two types of similarity and to examine their separate roles in analogical transfer.

\section{EXPERIMENT 2}

The purpose of Experiment 2 was to contrast the function of surface similarity with the role of procedural similarity in analogical problem solving. Surface similarity was manipulated by presenting either the abstract geometric shapes or the concrete line drawings of the elephant and rocks in the schematic pictures. The features of these objects were assumed to be irrelevant to both the general principle (the weight equivalence principle) and the procedural details. Procedural similarity, on the other hand, was manipulated by differentially depicting the ways to implement the weight equivalence principle, either consistently or inconsistently with the required target solution.

In addition to measuring subjects' generation of complete solutions to the target problem, subjects' attempts to use the general principle (i.e., a solution including the idea that the weight of the elephant can be compared to that of the smaller objects combined but with no complete procedure showing how to achieve this) were also measured. The major hypothesis was that if surface sim- 
ilarity enhanced access to the source principle, more subjects would attempt to use the principle (whether a general or a complete solution) in the similar-surface conditions than in the dissimilar-surface condition. Furthermore, if procedural similarity does indeed facilitate the execution of a principle, subjects in the similarprocedure conditions would generate complete solution more effectively and readily than those in the dissimilarprocedure condition.

\section{Method}

Subjects. One hundred and seven Unıversity of Kentucky undergraduate students who participated in this experiment for course credit were randomly assigned to six conditions. They were tested in groups of 15 to 21 .

Design, Materials, and Procedure. A 2 (surface features: similar, dissimilar) $\times 2$ (procedure: similar, dissimilar) between-subjects design was used in this study. The schematic pictures were created by manipulating both the surface features and the implementation procedure of the weight equivalence concept. Two pictures were similar to the target in implementation procedure, whereas the other two contained no similar procedure. The similar- and dissimilarprocedure pictures differed in procedural details in three ways: first, in the similar-procedure picture, the weights were added to a "floating" container rather than placed on a platform as in the dissımilar-procedure picture; second, in the similar-procedure picture, when a weight was added, the container sank farther into a pile of lines, whereas in the dissimilar-procedure picture, the platform was compressed; finally, in the similar-procedure picture, a large object was put in and then taken out of the container before the smaller items were placed in it. This differed from the dissimilarprocedure picture, in which the large and smaller items were placed side by side on two separate platforms.
The level of surface similarity depended on whether the source picture contained objects similar to those in the target problem. In the dissimilar-surface pictures, the big and smaller squares were included, whereas in the similar-surface pictures, the squares were replaced by sketches of an elephant and several rocks, which were attributionally similar to the key objects in the target problem. The four pictorial schematics are provided in Figure 2.

The target problem and line drawings of the relevant and irrelevant items with labels were identical to those used in Experiment 1 , except that a sketch of an elephant was added at the top of the target problem sheet and that the drawing of the boat used in Experiment 1 was replaced by another drawing of a boat, which looked different from the sketches of the container and lines in the similar-procedure pictures. The drawings of the elephant and boat can be seen in Appendix B. The experimental procedure was identical to that in Experiment 1.

Scoring. The scoring of the percentage of subjects successfully solving the target problem (complete solution) was the same as in Experiment 1. In addition, another measure was also scored to assess subjects' attempts to use the weight equivalence concept. For this measure, an answer that contained the general idea that smaller objects can be used to determine the elephant's weight because their combined weight equals that of the elephant received a score of 1 , regardless of whether the answer included concrete ways to implement this idea. If an answer included neither the general idea nor a concrete and complete procedure, a score of 0 was given.

\section{Results}

The percentage of subjects generating complete solutions across conditions is shown in Table 2 (column 2). The frequency of complete solutions was analyzed by a 2 (surface similarity) $\times 2$ (procedural similarity) ANOVA. The main effects of surface similarity $[F(1,103)=5.04$,

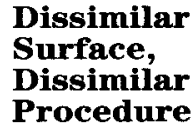

Similar

Surface,

Dissimilar

Procedure
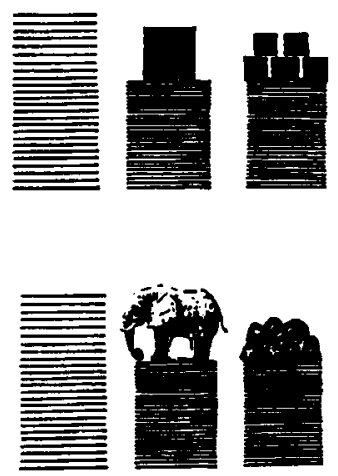
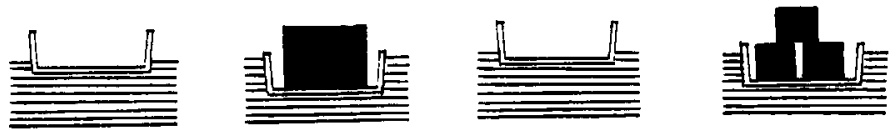

Similar

Procedure

Similar

Surface,

Similar

Procedure
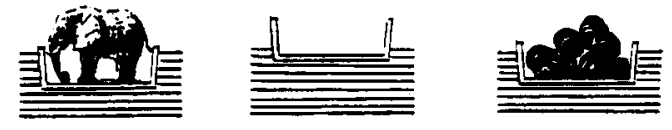

Figure 2. Schematic pictures used in Experiment 2. 
Table 2

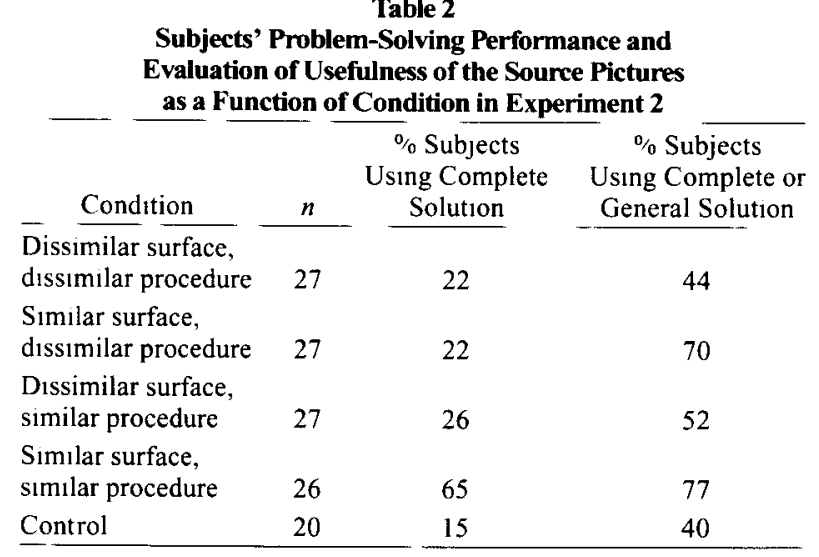

$\left.M S_{\mathrm{e}}=.199, p=.05\right]$ and procedural similarity $[F(1,103)$ $\left.=7.30, M S_{\mathrm{e}}=.199, p<.01\right]$ were significant. The interaction between these two factors was also significant $\left[F(1,103)=5.26, M S_{\mathrm{e}}=.199, p<.05\right]$, indicating that although both types of similarity facilitated solving the target problem, neither the surface nor the procedural similarity alone was sufficient for transfer. A StudentNewman-Keuls test revealed that subjects' proble-solving performance in the similar-surface, similar-procedure condition was reliably higher than in all other conditions ( $p s<.05)$. No other significant differences were obtained.

Table 2 also shows the percentage of subjects generating the general concept of weight equivalence and/or the complete solutions. A 2 (surface similarity) $\times 2$ (procedural similarity) ANOVA revealed a reliable main effect only of surface similarity $\left[F(1,103)=7.54, M S_{\mathrm{e}}=\right.$ $.229, p<.01]$. The main effect of procedural similarity was absent. The different patterns of these two dependent measures shed more light on the relations between surface and procedural similarity and the accessing and executing processes. Subjects in the similarsurface conditions accessed the weight equivalence concept and used the general idea more readily than those in the dissimilar-surface conditions. Yet the successful implementation of a complete solution depended on whether the source procedure was similar to the required target solution or not. Most subjects in the similarsurface, dissimilar-procedure condition experienced difficulty in implementing the general concept because of the discrepant procedure illustrated in the source picture. However, subjects in the dissimilar-surface, similarprocedure condition did not generate the complete solution more effectively than those in the dissimilarsurface, dissimilar-procedure condition, presumably because they failed to notice the analogous relationship between the source picture and the target problem.

\section{Discussion}

In this study, we made a preliminary attempt to distinguish two types of similarity and to identify two kinds of obstacles in the transfer process. One major finding is that although surface similarity did have a powerful effect on accessing an analogue, this type of similarity did not aid problem-solving performance when the procedural details illustrated in the analogue were not consistent with the target solution. Another finding is that the implementation procedure illustrated in the source pictures greatly influenced the transfer process; subjects who received a source picture with a similar procedure were better able to solve the target problem. A lack of common procedural details between the source analogue and the target problem apparently created an obstacle in transferring the conceptual information.

\section{EXPERIMENT 3}

Thus far, the results of the previous studies lend support to the assertion that procedural similarity between source pictures and a target problem facilitates the executing component in analogical problem solving. However, it is not yet obvious whether the manipulation of procedural similarity influences subjects' comprehension of the weight equivalence concept. Thus, one purpose of this study was to determine whether subjects' comprehension of the weight equivalence principle varied according to the particular schematic picture used in Experiment 2. Moreover, it is not evident whether the implementation procedure should be considered as one type of surface feature or whether it can be distinguished as an independent type of similarity. Some attempts have been made (e.g., Ross, 1989) to examine the different functions of superficial similarities in the analogical transfer process. Using mathematical problems, Ross found that surface similarity in object and story line affects the accessing process, while surface similarity in object correspondences between a source and a target problem have a pronounced effect on the use of a source solution. Experiment 3, then, was also designed to distinguish between these two types of similarity.

In this experiment, subjects were asked to interpret the conceptual meanings of the four source schematic pictures. They were also asked to rate the level of similarity in attributional (surface) features between each source picture and the standard picture, which contained the key objects of the target problem. If subjects interpreted the four source pictures in terms of the weight equivalence principle equally well and did not differentially rate the degree of surface similarity between the similarand dissimilar-procedure pictures, we would have better reason to conclude that the varied performance in Experiment 2 was due to the differing levels of similarity in implementation details between the source models and the target solutions, rather than to the degree of surface similarity.

\section{Method}

Subjects. The subjects were 38 undergraduate students at the University of Kentucky taking part in the experiment for course credit. None of them had participated in the previous studies. 
Design and Materials. This study contained two types of tasks for the subjects: one task involved interpreting the conceptual meaning of the four schematic pictures used in Experiment 2. The second task involved comparing the surface features of each schematic picture with the standard picture containing the drawings of an elephant, a boat, and rocks. (See Appendix B.)

Procedure. A booklet containing the schematic pictures and the comparison materials was introduced. The first page was the informed consent sheet, which notified subjects that they would be asked to interpret and compare some pictures. One of the four schematic pictures was included on the second page of the booklet, and one fourth of the subjects were asked to interpret the conceptual meaning of one of the four schematic pictures. This step took about $3 \mathrm{~min}$. On page 3 was the surface feature comparison task, for which subjects were told to compare the surface features of each of the four comparison pictures (labeled A, B, C, and D) with the standard picture ("How similar are these two pictures in terms of the shape of the objects?"). The attributional features of the objects were emphasized in order to minimize the impact of any possible structural and conceptual meanings. Before beginning this task, subjects were encouraged to scan all the pictures on the sheet and were informed that all the items in each picture should be taken into consideration while making comparisons. The rating of similarity between each comparison picture and the standard picture was based on a 7-point scale from 1 (not similar at all) to 7 (extremely similar). It took about $5 \mathrm{~min}$ to complete the comparison task.

\section{Results}

For the interpretation task, an explanation was considered correct if a subject's interpretation included the idea that the large item and the set of smaller objects weighed the same because they compressed down the platform to the same degree. The percentages of subjects providing correct interpretations for the dissimilarsurface, dissimilar-procedure; similar-surface, dissimilarprocedure; dissimilar-surface, similar-procedure; and similar-surface, similar-procedure picture were $66 \%$, $55 \%, 50 \%$, and $50 \%$, respectively. No significant differences were obtained, suggesting that all the schematic pictures depicted the weight equivalence concept equally effectively.

For the comparison task, subjects rated the similarsurface, similar-procedure and the similar-surface, dissimilar-procedure pictures as more similar to the standard picture (4.97 and 4.76, respectively) than the dissimilar-surface, similar-procedure and dissimilarsurface, dissimilar-procedure pictures (1.65 and 1.68, respectively). A 2 (surface features: similar, dissimilar) $\times 2$ (procedure: similar, dissimilar) within-subjects ANOVA was performed. Only the main effect of surface similarity was significant $\left[F(1,36)=382.39, M S_{\mathrm{e}}=\right.$ $.993, p<.0001]$. Paired comparisons revealed that the similarity ratings between the two similar-surface pictures were not reliably different, nor were those between the two dissimilar-surface pictures.

\section{Discussion}

Two major findings were obtained in this experiment. First, subjects interpreted the conceptual meanings of the four schematic pictures equally well, suggesting that the manipulation of either the surface similarity or the procedural similarity did not affect the ease with which the weight equivalence principle was comprehended. Thus, it is unlikely that the superior performance in the similar-procedure condition in Experiment 2 was due to the better comprehension of the general principle.

Second, the results of this study showed that subjects rated the surface similarity level between each similarsurface picture and the standard picture (e.g., the drawings of the elephant and rocks) overwhelmingly higher than that between each dissimilar-surface picture and the standard picture. Yet, the similar-procedure pictures were not rated as attributionally more similar to the standard picture than were the dissimilar-procedure pictures. Note that although the comparisons of the pictures in terms of the shape of the objects might have minimized the conceptual or procedural interference, subjects' comparisons may have focused more on the concrete objects (e.g., elephant and rocks) than on some less concrete ones (e.g., water-like lines) and, thus, the surface similarity between the similar- and dissimilar-procedure pictures might not have been precisely assessed. Nevertheless, the clear pattern of the subjects' ratings in surface similarity in this experiment suggests that the implementation procedure manipulated in Experiment 2 can be counted as a separate dimension of similarity and that it is therefore unlikely that the superior problemsolving performance in the similar-procedure conditions was due to a higher degree of surface-feature similarity in the former conditions than in the dissimilar-procedure conditions.

As a result of these experiments, we are prepared to assert that procedural similarity facilitates transfer by minimizing obstacles in executing the analogous concept. Yet, more direct evidence will be helpful in determining whether differences in transfer performance between the similar- and dissimilar-procedure conditions remain even when subjects are explicitly encouraged to use the source analogue.

\section{EXPERIMENT 4}

Experiment 4 was designed to investigate the nature of procedural similarity further. We employed a hint/nohint paradigm in order to provide additional evidence for the assertion that procedural similarity influences the execution component in transfer. As noted earlier in this article, the executing process plays a critical role in transfer and involves the implementation of a general principle or concept via a specific, concrete operational procedure. The obstacle encountered in transferring the source conceptual information can be explained, in part, as a consequence of the lack of procedural parallelism between the source analogues and the target problem. For even if subjects are able to encode and retrieve the conceptual meaning of the picture, they may not know how to apply it. 
For this experiment, then, the effects of similarity of implementation procedure on transfer were examined under the hint/no-hint conditions. It was hypothesized that adding similar implementation procedures to the source pictures would improve transfer performance, particularly when the subjects were instructed to use the source picture in solving the target problem. On the other hand, schematic pictures containing few common procedural details were predicted to remain inefficient even when subjects were explicitly told to consider using the source pictures.

\section{Method}

Subjects. Sixty-four University of Kentucky undergraduate students partıcipated in this experiment for course credit. Groups of 4 to 11 subjects were tested.

Design. A 2 (implementation procedure: similar, dissimilar) $\times$ 2 (Instruction: hint, no hint) between-subjects design was used in this experiment. Subjects in the dissimilar-procedure condition received source pictures which contained an implementation procedure different from that for the target solution. In the similarprocedure condition, subjects viewed a schematic picture illustrating the procedure required for the target problem. Neither picture included surface attributes highly similar to those of the target problem. Half the subjects in each condition received a hint to consider using the source picture before they attempted to solve the target; the other half received no such hint.

Materials and Procedure. The manipulation of the degree of procedural similarity was the same as in Experiment 2. In order to be sure that the level of attributional similarity between the similarprocedure picture and the target problem was not higher than that between the dissimilar-procedure picture and the target problem, the low-similarity picture of Experiment 1 was employed as the dissimilar-procedure picture and the dissimilar-surface, similarprocedure picture in Experiment 2 was used as the similar-procedure picture. These two schematics are presented in Figure 3.

The hint and no-hint conditions differed in the instructions given to the subjects. Subjects in the no-hint conditions were asked to view one of the source pictures carefully for $50 \mathrm{sec}$. Approximately 2 min elapsed between the picture-viewing and the problem-solving phases. The subjects were then asked to solve the target problem. The target problem was the same as in Experiment 1, and the procedure was the same as in the previous experiments.

The procedure and instructions in the hint conditions were the same as those in the no-hint conditions, with the exception that these subjects, after being introduced to the target problem, were informed that "the diagrammatic pictures you viewed a few minutes ago might help you to solve this problem." The subjects in the no-hint conditions received no such instruction.

\section{Results}

The scoring of subjects' problem-solving performance was identical to that in Experiment 2. The percentage of subjects successfully solving the target problem (generating a complete solution) in these four conditions is presented in Table 3 . A $2 \times 2$ ANOVA with procedure (similar, dissimilar) and hint (presence, absence) as the independent variables was performed. The outcome of this analysis revealed significant main effects for procedural similarity $[F(1,60)=6.16, p<.05]$ and for hint $[F(1,60)=5.08, p<.05]$. The interaction between these two factors was also marginally significant $[F(1,60)=$ $3.23, p=.07 ; M S_{\mathrm{e}}=.212$ for all the comparisons]. A Student-Newman-Keuls test revealed that the subjects' problem-solving performance in the similar-procedure, hint condition was reliably higher than in all other conditions $(p s<.05)$. No other significant differences were obtained. This pattern of performance shows that procedural similarity between the source pictures and the target problem along with the explicit hint to consider the source information facilitated subsequent transfer. More importantly, a hint to use the source picture was effective only when the pictorial schematics and the target problem shared a similar implementation procedure.

The frequency of attempts to use the general concept of weight equivalence is shown in Table 3 . A 2 (procedure: similar, dissimilar) $\times 2$ (hint: presence, absence) ANOVA was performed on this measure. The only reliable effect was that subjects in the hint conditions outperformed those in the no-hint conditions $[F(1,60)=$ $\left.10.63, M S_{\mathrm{e}}=.215, p<.001\right]$. The absence of the main effect for procedural similarity suggests that when the hint was provided, subjects in the dissimilar-procedure conditions experienced difficulty in implementing the general principle.

\section{Discussion}

What cognitive process is associated with the low transfer performance in the dissimilar-procedure conditions? The explanation emphasizing an obstacle in the execution of the principle is particularly convincing, given that in the no-hint conditions, subjects' performance in the dissimilar-procedure and similar-procedure conditions did not differ. However, once they were di-

Dissimilar Procedure

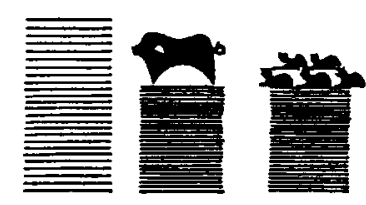

Similar Procedure
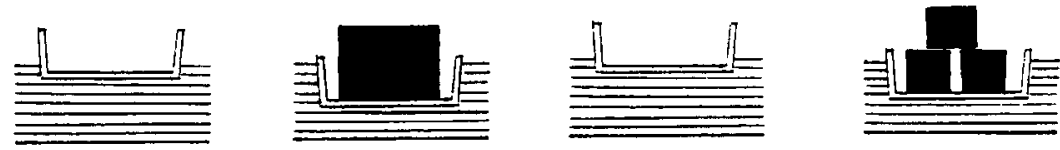

Figure 3. Schematic pictures used in Experiment 4. 
Table 3

Subjects' Problem-Solving Performance

as a Function of Condition in Experiment 4

\begin{tabular}{lccc}
\hline \multicolumn{1}{c}{ Condition } & $n$ & $\begin{array}{c}\text { \% Subjects } \\
\text { Using Complete } \\
\text { Solution }\end{array}$ & $\begin{array}{c}\text { \% Subjects } \\
\text { Using Complete or } \\
\text { General Solution }\end{array}$ \\
$\begin{array}{l}\text { Dissimilar procedure, } \\
\text { no hint }\end{array}$ & 15 & 27 & 40 \\
$\begin{array}{l}\text { Dissimilar procedure, } \\
\text { hint }\end{array}$ & 16 & 31 & 69 \\
$\begin{array}{l}\text { Similar procedure, } \\
\text { no hint }\end{array}$ & 17 & 35 & 41 \\
$\begin{array}{l}\text { Similar procedure, } \\
\text { hint }\end{array}$ & 16 & 81 & 88 \\
\hline
\end{tabular}

rected to access and use the source pictures, more subjects in the similar-procedure condition generated correct solutions than in the dissimilar-procedure condition. In the similar-procedure, hint condition, most subjects who came up with the general solution also generated a complete, workable solution, whereas in the dissimilarprocedure, hint condition, only about half the subjects who used the general solution also executed a complete procedure.

The current data indicate that both the weight equivalence principle and the buoyancy idea (the boat solution procedure) are important to the generation of an appropriate and complete solution. The principle alone is not sufficient for producing transfer because of its lack of procedural specificity (i.e., its lack of a suggestion to use the boat). On the other hand, if the idea of buoyancy is isolated from the general solution principle, the buoyancy concept is also inefficient in promoting problem solving, an argument supported by the fact that many subjects failed to generate complete solutions even when they attempted to use the boat (e.g., "put the elephant in the boat and see whether the boat sinks").

The present experiment demonstrated the effects of procedural similarity on transfer via the executing component. Yet, because the perceptual attributes of the two schematic pictures were not identical, it could be argued that these differential perceptual attributes also influenced the transfer process. Therefore, a group of 31 subjects was asked to determine which of the two source pictures was more similar in superficial attributes to the key items used in the target problem. Fifty-five percent of the subjects chose the dissimilar-procedure picture as more similar to the target items, whereas $45 \%$ of them chose the similar-procedure picture, indicating that the two source pictorial analogues were viewed as equally similar (or dissimilar) in surface features to the key items of the target problem. These results suggest that the superior transfer performance obtained in the similarprocedure condition was not due to attributional similarity. These findings distinguish similar implementation procedure from similar surface features, lending support to our original hypothesis that the implementation procedure, a feature distinct from superficial attrib- utes, has a powerful impact on the executing process of transfer.

\section{GENERAL DISCUSSION}

\section{Transferring Conceptual Information From Pictures to Problems}

Some previous studies have shown that people who receive a visual representation do not benefit from it when solving an analogous problem (e.g., Gick, 1985), whereas others have demonstrated the facilitative effects of pictorial analogues in problem solving (e.g., Beveridge \& Parkins, 1987; Dreistadt, 1969). We attempted to go beyond the issue of whether pictorial analogues aid problem solving and to investigate instead the source of variation in the use of visual analogues by considering the following central questions: What properties of a picture facilitate solving analogous problems? And in what ways does similarity between a source picture and a target problem influence the accessing and executing processes in transfer?

Our results in the present research begin to elucidate the conditions under which solvers might have difficulty in spontaneously accessing a pictorial analogue and also experience obstacles in executing a learned solution principle. A specific effort was made to distinguish between two types of similarity which are theorized to affect the accessing and executing processes. The present research demonstrates that low commonalities in surface features and procedural details are two major reasons why subjects may have difficulty in retrieving the relevant information and in executing the solution principle. As with verbal analogies, whether a schematic picture can serve as an efficient analogue in problem solving depends on how the analogous information or solution principle is illustrated. In the following two sections, we delineate the relations between two types of similarity and two of the cognitive processes of analogical transfer.

\section{Types of Similarity and Processes of Transfer}

Surface similarity and the accessing process. One reason for ineffectiveness in the transfer of a schematic picture may be that it provides inadequate attributional cues for retrieving the analogous relationship when solving a target problem. Consistent with previous studies of verbally based analogy, the present research reveals that spontaneous analogical transfer in problem solving is more likely to be achieved when a target problem and a source schematic picture share surface (physical) features. A picture containing attributes similar to those in the target problem may remind problem solvers of the source analogue and thus serve as a vehicle for retrieving the essence of a schematic picture.

However, the results of the present experiments also indicate that surface similarity does not automatically aid transfer performance. When the schematic picture and the target problem shared only a general principle but not an operational procedure, surface similarity did not 
enhance subjects' generation of complete solutions. The problem-solving performance patterns in Experiments 2 and 4 are very similar, suggesting that similarity in surface features plays a role similar to that of a hint in the retrieval of a source analogue, but that neither surface similarity nor a hint affects how the source principle is applied or implemented.

Procedural similarity and the executing process. The present findings clearly reveal the importance of another type of similarity-specifically, procedural details shared by a source picture and a target problem - for analogical problem solving. Subjects receiving source pictures with an implementation procedure similar to the required target solution transferred the analogous information more readily and successfully. In contrast, subjects encountered considerable difficulty in using an analogous principle when the source pictures did not contain a detailed procedure for implementing the general concept.

These results indicate that the absence of a similar implementation procedure in a schematic picture is, in part, responsible for lack of transfer. But can the effective transfer resulting from the presence of a similar implementation procedure be interpreted as resulting from other types of similarity? The answer appears to be no. This dimension of similarity was shown to be distinguishable from other types of similarity. First, the level of procedural similarity was manipulated independently of the essential conceptual similarity. Both the similar- and the dissimilar-procedure pictures depicted the same general principle (weight equivalence) but illustrated different concrete ways to achieve this principle. Moreover, the implementation procedure is not related to surface similarity, as is evidenced by the results of Experiment 3, wherein subjects did not view the similar-procedure pictures as attributionally more similar to the target items than the dissimilar-procedure pictures.

What cognitive component is responsible for the effects of procedural similarity on transfer? As defined earlier in this article, the executing component involves implementing a general principle, rule, concept, or idea in a specific context with a concrete operational procedure. The data from the present experiments clearly indicate that the degree of similarity of implementation procedure affects transfer via the executing process. This interpretation seems particularly convincing for several reasons. First, in Experiments 2 and 4, similar surface features and a hint did not enhance transfer performance in the dissimilar-procedure conditions, suggesting that those subjects experienced difficulty in applying the solution rather than in retrieving the relevant information. If the obstacle was in the retrieving process, either surface similarity or a hint that explicitly encouraged the subjects to use the information from the source picture should have facilitated transfer performance.

More evidence supporting the executing process explanation is that once surface similarity was added or when the subjects were instructed to use the source pictures, subjects in the dissimilar-procedure conditions attempted to use the general principle but failed to achieve complete solutions. In contrast, most subjects in the similar-procedure conditions successfully executed the solution; their answers included appropriate, concrete ways to operationalize the general principle. Clearly, the similar procedure guided the subjects in implementing the analogous solution for the target problem. Thus, these findings support our conclusion that implementational similarity facilitates the executing process.

The present findings concerning the executing component are consistent with recent studies (e.g., Novick \& Holyoak, 1991) of mathematical problem solving. Novick and Holyoak demonstrated that when numerical correspondences between source and target problems were clearly specified, students were more likely to transfer an appropriate mathematical procedure successfully than when only conceptual relationships were provided. The authors accounted for the benefit of the numerical correspondences in terms of an enhanced ability to adapt the source solution procedure to the target problem. Other researchers (e.g., Anderson et al., 1984; Medin \& Ross, 1989; Reed \& Bolstad, 1991; Smith, Langston, \& Nisbett, 1992) have also concluded that general instructions are often less effective than concrete instances in learning a task, because abstract information typically fails to provide a procedure for performing the task.

\section{Implications and Limitations of the Present Findings}

The specifications between types of similarity and their associations with different cognitive processes have important implications for future research in the field of analogical problem solving. The present results extend a broad picture of the conditions that govern analogical problem solving. It is evident in the present research that the overall relations between source and target problems are multidimensional and involve several types of similarity and that the cognitive activities associated with analogical transfer are often multicomponential, involving several effortful processes. A fruitful path of further research may involve further examination of the circumstances under which efficient pictorial transfer can be achieved and exploration of precisely how individuals come to understand, retrieve, and apply the solution principle from pictures in problem solving.

The present findings may be valuable in explaining why some pictures can serve as powerful analogues and produce robust transfer whereas others do not. For example, the lack of transfer from the diagram used in Gick's research (1985) may be due to an accessing problem because of the low surface resemblance between the diagram and the verbally based target problem and/or to an obstacle in the execution component. The results obtained in the present research on the effects of the various types of similarity on transfer may prove applicable not only to visually based analogical transfer but to learning and problem solving in general. For example, one common finding in the learning and problem-solving literature is that externally provided abstract rules, principles, or concepts often fail to be effective for subse- 
quent problem solving (see, e.g., Anderson et al., 1984; Brown \& Kane, 1988; LeFevre \& Dixon, 1986). It has not yet been clearly demonstrated why such principles tend to be inert in transfer. The present findings suggest that the absence of a similar implementation procedure may be responsible for transfer inadequacy.

It needs to be pointed out that the present research is only a preliminary exploration of the effects of pictorial analogues on problem solving. Undoubtedly, much about how pictorial analogues are used in learning and problem solving remains unexplored and certain limitations of the current findings need to be overcome. First, the data obtained in this research were based on only a restricted insight problem situation, and thus, the particular features of this type of problem might have constrained the patterns of transfer performance. Employing various types of problems (e.g., ill-defined and well-defined problems) in examining pictorial transfer will improve the generalizability of the conclusions drawn in the present studies.

Moreover, investigations of the mechanisms underlying pictorial transfer should not be limited to manipulations of the features of pictorial schematics. In order to understand precisely what characteristics of pictures affect the transfer process, the functions of different types of pictures in problem solving need to be compared. For example, it may be valuable to examine the degree to which a schematic picture produces benefits in problem solving relative to a realistic drawing or even a dynamic visual display. Also, although the present data lend support to the assumption that the same principles govern pictorially and verbally based analogical reasoning, a better understanding of the similarities and differences between the analogical functions of verbal and pictorial representations may require direct comparisons of these different modalities.

In conclusion, the present research is an initial step toward a systematic investigation of problem solving by pictorial analogy. Although a vast body of research has indicated the powerful function of language-based analogy in problem solving, the present research clearly demonstrates that analogical problem solving is not restricted to the transfer of verbal analogues and provides tentative support for the hypothesis that similar mechanisms operate on both verbal and pictorial analogical transfer. The present results indicate that problem solving by pictorial analogy, as by verbal analogy, involves complex cognitive processes: the conceptual meaning of a schematic picture needs to be extracted and encoded; the analogous information needs to be retrieved; and the concepts or general solutions need to be executed. Further clarification of the effects of different modalities of source analogues, different types of pictorial displays, and different pictorial features on the transfer will advance our understanding of pictorial information processing and will have significant implications for education.

\section{REFERENCES}

Anderson, J. R., Farrel.l, R., \& Sauers, R. (1984). Learning to program in L.ISP. Cognitive Science, 8, 87-129.
BassoK, M. (1990). Transfer of domain-specific problem-solving procedures. Journal of Experimental Psychology. Learning, Memory, \& Cognition, 16, 522-533.

BASSOK, M., \& HolyOAK, K. J. (1989). Interdomain transfer between isomorphic topics in algebra and physics. Journal of Experimental Psychology. Learning, Memory, \& Cognition, 15, 153-166.

Bevan, M. F., Denton, J. Q., \& Myers, J. L. (1974). The robustness of the $F$ test to violations of continuity and form of treatment populations. British Journal of Mathematical \& Statistical Psychology, 27, 199-204.

BeVeridge, M., \& Parkins, E. (1987). Visual representation in analogical problem solving. Memory \& Cognition, 15, 230-237.

Brown, A. L., \& Campione, J. C. (1981). Inducing flexible thinking: A problem of access. In M. Freidman, J. P. Das, \& N. O'Connor (Eds.), Intelligence and learning (pp. 515-530). New York: Plenum.

Brown, A. L., \& CAMPIONE, J. C. (1984). Three faces of transfer: Implications for early competence, individual differences, and instruction. In M. Lamb, A. Brown, \& B. Rogoff (Eds.), Advances in developmental psychology (Vol. 3, pp. 143-192). Hillsdale, NJ: Erlbaum.

Brown, A. L., \& Kane, M. J. (1988). Preschool children can learn to transfer: Learning to learn and learning from example. Cognitive Psychology, 20, 493-523.

Brown, A. L., Kane, M. J., \& Echols, C. H. (1986). Young children's mental models determine analogical problems with a common goal structure. Cognitive Development, 1,103-121.

Chafe, W. L. (1976). Creativity in verbalization as evidence for analogical knowledge. In R. C. Schank \& B. L. Nash-Webber (Eds.), Theoretical issues in natural language processing (pp. 158-159). Cambridge, MA: Bolt, Beranek, \& Newman.

Chen, Z., \& Daehler, M. W. (1989). Positive and negative transfer in analogical problem solving by 6-year-old children. Cognitive $\mathrm{De}$ velopment, 4, 327-344.

Chen, Z., \& Daehler, M. W. (1992). Intention and outcome: Key components of causal structure facilitating mapping in children's analogical transfer. Journal of Experimental Child Psychology, 53, 237-257.

Clement, C. A. (1994). Effect of structural embedding on analogical transfer: Manifest versus latent analogs. American Journal of Psychology, 107, 1-38.

Clement, C. A., \& Gentner, D. (1991). Systematicity as a selection constraint in analogical mapping. Cognitive Science, 15, 89-132.

Crisafi, M. A., \& Brown, A. L. (1986). Analogical transfer in very young children: Combining two separately learned solutions to reach a goal. Child Development, 57, 953-968.

DAeHLER, M. W., \& Chen, Z. (1993). Protagonist, theme, and goal object: Effects of surface features on analogical transfer. Cognitive Development, 8, 211-229.

DREISTADT, R. (1969). The use of analogies and incubation in obtaining insights in creative problem solving. Journal of Psychology, 71, 159-175.

DunCKer, K. (1945). On problem solving. Psychological Monographs, 58, (5, No. 270).

GENTNER, D. (1989). The mechanisms of analogical learning. In S. Vosniadou \& A. Ortony (Eds.), Similarity and analogical reasoning (pp. 199-241). Cambridge: Cambridge University Press.

GiCK, M. L. (1985). The effect of a diagram retrieval cue on spontaneous analogical transfer. Canadian Journal of Psychology, 39, 460-466.

Gick, M. L., \& HolyoaK, K. J. (1980). Analogical problem solving. Cognitive Psychology, 12, 306-335.

Gick, M. L., \& HolyoaK, K. J. (1983). Schema induction and analog1cal transfer. Cognitive Psychology, 15, 1-38.

Gordon, W. J. J. (1961). Synectics. New York: Harper.

Gordon, W. J. J. (1979). Some source material in discovery-by-analogy. Journal of Creative Behavior, 8, 239-257.

HolyoAK, K. J. (1984). Analogical thinking and human intelligence. In R. J. Sternberg (Ed.), Advances in the psychology of human intelligence (Vol. 2, pp. 199-230). Hillsdale, NJ: Erlbaum.

Holyoak, K. J., Junn, E. N., \& Billman, D. O. (1984). Developmental analogical problem-solving skill. Child Development, 55, 2042-2055.

HoI YOAK, K. J., \& KOH, K. (1987). Surface and structural similarity in analogical transfer. Memory \& Cognition, 15, 332-340.

Hsu, T. C., \& FELdT, L. S. (1969). The effect of limitations on the num- 
ber of criterion score values on the significance of the $F$ test. American Educational Research Journal, 6, 515-527.

Johnson, M. K., Bransford, J. D., Nyberg, S. E., \& Cleary, J. J. (1972). Comprehension factors in interpreting memory for abstract and concrete sentences. Journal of Verbal Learning \& Verbal Behavior, 11, 451-454.

KINTSCH, W. (1974). The representation of meaning in memory. Hillsdale, NJ: Erlbaum.

KRUEGER, T. (1981). Imagery pattern in creative problem solving. Las Cruces, NM: Encina Press.

LARKIN, J. H., \& SimON, H. A. (1987). Why a diagram is (sometimes) worth ten thousand words. Cognitive Science, 11, 65-99.

LeFevre, J. A., \& Dixon, P. (1986). Do written instructions need examples? Cognition \& Instruction, 3, 1-30.

LEVIN, J. R. (1982). Pictures as prose-learning devices. In A. Flammer $\&$ W. Kintsch (Eds.), Discourse processing (pp. 412-444). Amsterdam: North Holland.

MAYER, R. E., \& GALLINI, J. K. (1990). When is an illustration worth ten thousand words? Journal of Educational Psychology, 82, 715-726.

MEDIN, D. L., \& Ross, B. H. (1989). The specific character of abstract thought: Categorization, problem solving, and induction. In R. J. Sternberg (Ed.), Advances in the psychology of human intelligence (Vol. 5 , pp. 189-223). Hillsdale, NJ: Erlbaum.

MYERS, J. L., \& WeLL, A. D. (1991). Research design and statistical analysis. New York: Harper Collins.

Needham, D. R., \& BegG, I. M. (1991). Problem-oriented training promotes spontaneous analogical transfer: Memory-oriented training promotes memory for training. Memory \& Cognition, 19, 543-557.

Novick, L. R. (1988). Analogical transfer, problem similarity, and expertise. Journal of Experimental Psychology: Learning, Memory, \& Cognition, 14, 510-520.

NovicK, L. R. (1990). Representational transfer in problem solving. Psychological Research, 1, 128-132.

Novick, L. R., \& HoLYOAK, K. J. (1991). Mathematical problem solving by analogy. Journal of Experimental Psychology: Learning, Memory, \& Cognition, 17, 398-415.

OPPENHEIMER, J. R. (1956). Analogy in science. American Psychologist, 11, 127-135.

Perfetto, G. A., Bransford, J. D., \& Franks, J. J. (1983). Constraints on access in a problem solving context. Memory \& Cognition, 11, 24-31.

PezdeK, K., \& Royer, J. M. (1974). The role of comprehension in learning concrete and abstract sentences. Journal of Verbal Learning \& Verbal Behavior, 13, 551-558.

REED, S. K. (1987). A structure-mapping model for word problems. Journal of Experimental Psychology: Learning, Memory, \& Cognition, 13, 124-139.

REED, S. K. (1989). Constraints on the abstraction of solution. Journal of Educational Psychology, 81, 532-540.

REED, S. K., \& BolSTAD, C. A. (1991). Use of examples and procedures in problem solving. Journal of Experimental Psychology: Learning, Memory, \& Cognition, 17, 753-766.

Ross, B. H. (1984). Remindings and their effects in learning a cognitive skill. Cognitive Psychology, 16, 371-416.

Ross, B. H. (1987). This is like that: The use of earlier examples and the separation of similarity effects. Journal of Experimental Psychology: Learning, Memory, \& Cognition, 13, 629-639.

Ross, B. H. (1989). Distinguishing types of superficial similarities: Different effects on access and use of earlier problems. Journal of Experimental Psychology: Learning, Memory, \& Cognition, 15, 456468.

Ross, B. H., \& KENNEDY, P. T. (1990). Generalizing from use of earlier examples in problem solving. Journal of Experimental Psychology: Learning, Memory, \& Cognition, 16, 42-55.

Ross, B. H., RYAN, W. J., \& TENPENNY, P. L. (1989). The access of relevant information for solving problems. Memory \& Cognition, 17, 639-651.

SIMON, H. A. (1981). The sciences of the artificial. Cambridge, MA: MIT Press.

Smith, E. E., Langston, C., \& Nisbett, R. E. (1992). The case for rules in reasoning. Cognitive Science, 16, 1-40.

Stein, B. S., Way, K. R., Benningfiel.d, S. E., \& Hedgecough, C. A.
(1986). Constraints on spontaneous transfer in problem-solving tasks. Memory \& Cognition, 14, 432-441.

VosNiadou, S. (1989). Analogical reasoning as a mechanısm in knowledge acquisition: A developmental perspective. In S. Vosniadou \& A. Ortony (Eds.), Similarity and analogical reasoning (pp. 438469). Cambridge: Cambridge University Press.

Weisberg, R. W., DiCamillo, M., \& Phillips, D. (1978). Transferring old associations to new problems: A non-automatic process. Journal of Verbal Learning \& Verbal Behavior, 17, 219-228.

Wertheimer, M. (1959). Productive thinking. New York: Harper \& Row.

\section{NOTE}

1. Because the efficiency scores measuring subjects' problem-solving performance were highly consistent with the percentage of subjects successfully solving the target problem in all three studies, the efficiency scale is not considered further in our discussion of the results in each experiment.

\section{APPENDIX A}

The Target Story Problem and an Example of the Item Sketches Used in the Present Research

"Weigh the Elephant" Problem:

Many years ago there lived in China a young man. Wishing to further his education, he went to a wise man in a remote land.
Table

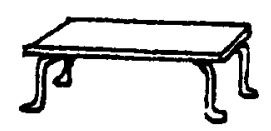

Boat

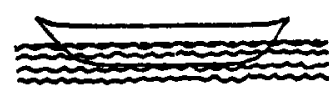

Rocks

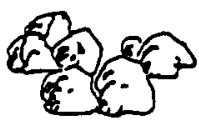

Containers

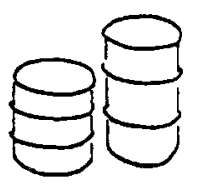

Animals

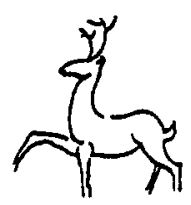

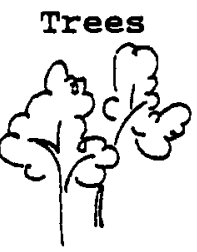

Small Scale

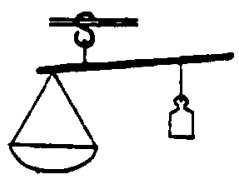

Boxes

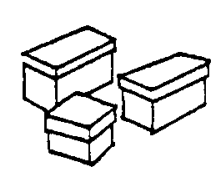

Figure A1. An example of the items for the target problem. 
"Master," he said, "if you will allow me to study with you for one year, I will give you, in payment, this elephant." And he displayed to the wise man an elephant, strong and beautiful.

The old man looked from the young man to the elephant, and asked: "How much does the elephant weigh?"

"I do not know, Master," the boy replied.

"Weigh the elephant. Come back tomorrow and we will begin to learn from each other."

So the boy left, running through the town, looking for a scale to weigh the elephant. The largest scale he could find, however, was only scaled to 200 pounds.

The next morning the boy sat, despondent, under a big tree, on a rocky river bank. As he watched, a boat came into view; the old man was rowing toward him. The old man got out of the boat, went to the boy and sat down.

"How much does your elephant weigh?"
"I cannot find a large scale, Master."

"It is not the elephant I am measuring, my son. It is the student's thinking. You have everything you need to weigh the elephant. When you have done so, you may join me." And the old man stood up and moved up the path to his school, leaving the boy with the problem.

\section{APPENDIX B}

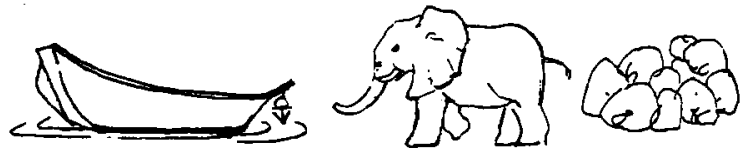

Figure B1. The standard picture used in Experiment 3. 